\title{
Trauma complexo e suas implicações diagnósticas
}

\author{
Complex trauma and diagnostic implications
}

\author{
Thiago Wendt Viola ${ }^{1}$, Bruno Kluwe Schiavon ${ }^{2}$, Anelise Meurer Renner ${ }^{3}$, Rodrigo Grassi-Oliveira ${ }^{4}$
}

${ }^{1}$ Acadêmico de Psicologia, Pontifícia Universidade Católica do Rio Grande do Sul (PUCRS), Porto Alegre, RS. Bolsista, Iniciação Científica, Conselho Nacional de Desenvolvimento Científico e Tecnológico (CNPq), Grupo de Pesquisa em Neurociência Cognitiva do Desenvolvimento (GNCD), Programa de Pós-Graduação em Psicologia: Cognição Humana, PUCRS. ${ }^{2}$ Acadêmico de Psicologia, PUCRS. Bolsista, Iniciação Científica, Bolsa de Pesquisa para Alunos da Graduação (BPA) da PUCRS, GNCD, Programa de Pós-Graduação em Psicologia: Cognição Humana, PUCRS. ${ }^{3}$ Acadêmica de Psicologia, PUCRS. Auxiliar de Pesquisa, GNCD, Programa de PósGraduação em Psicologia: Cognição Humana, PUCRS. ${ }^{4}$ MD, MSc, PhD. Professor adjunto, Programa de Pós-Graduação em Psicologia: Cognição Humana, PUCRS.

Suporte financeiro: Conselho Nacional de Desenvolvimento Científico e Tecnológico (CNPq) e Bolsa de Pesquisa para Alunos da Graduação da Pontifícia Universidade Católica do Rio Grande do Sul (BPA-PUCRS).

\section{Resumo}

Aexposição prolongada a múltiplos eventos traumáticos de natureza interpessoal, sobretudo durante o desenvolvimento, tem demonstrado consequências e sintomas psiquiátricos não considerados pelo atual diagnóstico de transtorno de estresse pós-traumático (TEPT). Essas situações negativas e crônicas durante a infância e adolescência evidenciam a existência de uma síndrome psicopatológica associada a complexas (des)adaptações a efeitos traumáticos diversos. A ausência de um diagnóstico coeso e fidedigno para essas vítimas interfere negativamente na identificação sintomatológica e no método de tratamento. O presente trabalho visa revisar a definição de trauma, apresentando o conceito de trauma complexo, explorando suas implicações clínicas, bem como as categorias diagnósticas derivadas desse constructo. Importantes questões são levantadas acerca das diferenças entre trauma complexo e TEPT, investigando os sintomas e transtornos comórbidos ao diagnóstico de TEPT, assim como as limitações inerentes a esse diagnóstico. Considerando o impacto psicopatológico relacionado ao trauma complexo, discute-se a possível inserção de uma nova categoria diagnóstica na $5^{\text {a }}$ versão do Manual Diagnóstico e Estatístico dos Transtornos Mentais, adjunta ao espectro de psicopatologias pós-traumáticas. Descritores: Transtornos de estresse pós-traumáticos, psicopatologia, estresse psicológico, transtornos traumáticos cumulativos.

\begin{abstract}
Prolonged exposure to multiple traumatic events of an interpersonal nature, particularly during development, has shown psychological consequences and symptoms not included among the current diagnostic criteria of post-traumatic stress disorder (PTSD). These negative and chronic situations during childhood and adolescence provide further evidence of the existence of a psychopathological syndrome associated with complex (dis)adaptations to a number of traumatic effects. The absence of a cohesive and reliable diagnosis for these patients negatively affects symptom identification and treatment planning. The aim of the present study was to review the definition of trauma, presenting the concept of complex trauma and investigating its clinical implications and the diagnostic categories deriving from this construct. Important questions are raised about differences between complex trauma and PTSD, followed by an investigation of PTSD symptoms and comorbid disorders, as well as the limitations of PTSD diagnosis. Taking into consideration the psychopathological impact associated with complex trauma, the possibility of including a new diagnostic category in the 5th edition of the Diagnostic and Statistical Manual of Mental Disorders is discussed, as part of the spectrum of post-traumatic psychopathologies. Keywords: Post-traumatic stress disorders, psychopathology, psychological stress, cumulative trauma disorders.
\end{abstract}

\footnotetext{
Correspondence: E-mail: rodrigo_grassi@terra.com.br

Não foram declarados conflitos de interesse associados à publicação deste artigo.

Copyright (C) Revista de Psiquiatria do Rio Grande do Sul - APRS
}

Rodrigo Grassi-Oliveira, Grupo de Pesquisa em Neurociência Cognitiva do Desenvolvimento (GNCD), Faculdade de Psicologia, Pontifícia Universidade Católica do Rio Grande do Sul, Av. Ipiranga, 6681, prédio 11, sala 936, Partenon, CEP 90619-900, Porto Alegre, RS, Brasil. Tel.: (51) 3320.3550, ramal 7740. Fax: (51) 3320.3633.

Recebido em 20/11/2010. Aceito em 02/12/2010. 


\section{Introdução}

Considerando o grande progresso nos estudos de trauma psicológico, a validade dos critérios diagnósticos do transtorno de estresse pós-traumático (TEPT) é cada vez mais discutida. O critério A descrito na $4^{\mathrm{a}}$ edição do Manual Diagnóstico e Estatístico dos Transtornos Mentais (DSM-IV) ${ }^{1}$ é o principal alvo das críticas, especialmente no que tange ao conceito do que seria trauma $a^{2,3}$. Ao contrário dos outros transtornos psiquiátricos, o TEPT possui um critério etiológico específico: a exposição ao critério A é considerado o principal fator para a desordem, sugerindo a existência de um critério-chave que implica uma relação única entre o trauma e o diagnóstico de TEPT. No entanto, alguns autores afirmam que, até o momento, não há uma definição precisa de quais estressores seriam capazes de produzir sintomas de TEPT, principalmente em virtude de alguns estudos terem comprovado que: a) os sintomas de TEPT são amplamente encontrados em reações a eventos estressores que não se enquadrariam no critério $\mathrm{A}$; e b) eventos traumáticos nem sempre estão associados ao TEPT, podendo estar associados ao desenvolvimento de sintomas de outros transtornos psiquiátricos $^{2,4}$.

Por outro lado, outros autores sugerem que o diagnóstico atual de TEPT não contemplaria todos os efeitos decorrentes da exposição a eventos traumáticos durante o desenvolvimento. Nesse contexto, alguns autores têm proposto o conceito de trauma complexo como alternativa para descrever aqueles eventos traumáticos cuja exposição é múltipla, crônica e prolongada ${ }^{5}$. Considerando a carência de estudos no Brasil sobre essa temática e uma possível reestruturação dos critérios diagnósticos para TEPT na $5^{\mathrm{a}}$ edição do DSM (DSM-V), este artigo é atual e visa apresentar e descrever o conceito de trauma complexo e suas implicações diagnósticas.

\section{Trauma e TEPT}

Com o fim da guerra no Vietnã, muitos combatentes retornaram ao seu país de origem apresentando inúmeras queixas relacionadas às lembranças recorrentes de suas vivências traumáticas intensas. Tal situação exigiu a criação de uma categoria diagnóstica que compreendesse as consequências da exposição à guerra e a outros eventos traumáticos, o que culminou com a inclusão do TEPT na $3^{a}$ edição do DSM (DSM-III) $)^{6}$. Na concepção inicial do DSM-III, evento traumático foi definido como um estressor catastrófico fora do escopo de experiências esperadas para a vida de alguém. Os responsáveis pelo consenso diagnóstico do TEPT no contexto do DSM-III tinham em mente eventos como guerra, tortura, estupro, holocausto, as bombas atômicas de Hiroshima e Nagasaki, assim como desastres naturais (terremotos, furacões, etc.) e outros acidentes (explosões de fábricas, acidentes de avião e automobilísticos, etc.). Esses autores consideravam que eventos traumáticos eram claramente diferentes de estressores muito dolorosos mas esperados, que constituem vicissitudes normais da vida, como divórcio, rejeição, doença grave e assim por diante. Assim, se algum sintoma fosse observado posteriormente a esses eventos, o diagnóstico correto deveria ser o de transtorno de ajustamento. Essa dicotomização entre o que seria traumático e o que não seria foi baseada no pressuposto de que a maioria dos indivíduos consegue lidar com o estresse comum, mas sua capacidade adaptativa é insuficiente frente a um evento inesperado e catastrófico.

Considerando a estruturação diagnóstica inicial do TEPT descrita no DSM-III, acumularam-se evidências de que alguns eventos estressores poderiam ser desencadeados por situações da vida cotidiana e poderiam produzir efeitos comparáveis ao estresse gerado pelas vivências de guerra, por exemplo. Assim, alguns critérios diagnósticos do TEPT foram revisados e reformulados para o DSM-IV, levando em conta que a resposta ao estresse é altamente variável. Desse modo, ampliou-se a definição de quais eventos estressores estariam associados ao TEPT $^{7}$. Um critério sobre a subjetividade do evento passou a ser considerado. No DSM-IV, o critério A, que define a etiologia do TEPT, passou a ser composto por dois componentes principais: um definindo as características da vivência traumática (critério A1: vivência, testemunho ou confronto de eventos que envolvam ameaça à integridade física própria ou de outros) e outro caracterizando a resposta da vítima a tal evento (critério A2: resposta do indivíduo deve envolver intenso medo, impotência ou horror $)^{8}$.

Ressalta-se que, desde sua primeira concepção, os sintomas apresentados no diagnóstico de TEPT devem estar associados ao evento traumático estressor, tornando o critério A fundamental na definição etiológica do transtorno9. Entretanto, frente às críticas sugeridas em torno da definição do critério A do TEPT, o comitê responsável pelo DSM-V pretende novamente reestruturar os critérios diagnósticos do transtorno, partindo da expansão do critério A1 e da retirada do critério A2 (características da resposta ao estresse) ${ }^{10,11}$.

Ao mesmo tempo, visando compreender padrões complexos e consistentes de distúrbios psicológicos associados à exposição contínua a eventos traumáticos, alguns autores iniciaram um novo campo de estudo adjunto às discussões referentes ao estresse pós-trauma. Ainda no desenvolvimento do DSM-IV, a American Psychiatric Association (APA) organizou um estudo com 528 participantes, a saber, adolescentes e adultos com histórico de exposição a eventos traumáticos ou subtraumáticos. Esse trabalho de campo teve o intuito de propor algumas alterações no diagnóstico de TEPT, explorando o impacto da exposição crônica ao trauma ${ }^{12}$.

Baseado nesses estudos e em experiências clínicas, um questionamento no sentido de que o diagnóstico de TEPT muitas vezes não seria o mais adequado em casos de eventos estressores crônicos começou a ganhar espaço na comunidade especializada. A definição de trauma pelo DSM-IV não foi considerada apropriada em muitos casos, principalmente 
quando os efeitos pós-traumáticos são observados em crianças e adolescentes ${ }^{13}$. Partindo do conceito de trauma complexo, alguns autores propuseram a criação de uma nova categoria diagnóstica relacionada a sintomas pós-traumáticos: o transtorno de estresse extremo sem outra especificação (disorders of extreme stress, not otherwise specified - DESNOS $)^{14,15}$. Apesar de o DESNOS ter representado um avanço significativo nos estudos de trauma cumulativo, o comitê organizador do DSM-IV optou pela não inserção de uma nova categoria diagnóstica única, justificando sua decisão pela necessidade de mais estudos. Após mais de uma década, outro grupo de autores, considerando os estudos realizados sobre o DESNOS e também sobre os efeitos do trauma ao longo do desenvolvimento, principalmente estudos realizados com crianças, propôs outra categoria diagnóstica, denominada de transtorno de desenvolvimento traumático (developmental trauma disorder - DTD $)^{16}$. No presente momento, o comitê organizador da APA estuda a possibilidade de inclusão de tais categorias (DESNOS e DTD) no DSM-V, cujo lançamento está previsto para 2012.

\section{Trauma complexo}

As reações e consequências ao trauma diferem quanto aos tipos de eventos traumáticos. Em um extremo, há os indivíduos de idade adulta que sofreram um incidente único (acidente de automóvel, um assalto, um estupro, etc.); no lado oposto estão as respostas ao trauma de início precoce, de ocorrências múltiplas e, às vezes, de natureza invasiva e interpessoal (maus-tratos na infância, negligência infantil, violência doméstica, etc. $)^{17}$. O impacto de eventos traumáticos isolados é bem descrito no diagnóstico de TEPT, porém existe uma vasta literatura evidenciando que experiências prolongadas adversas na infância, como abuso e negligência, estão associadas a agravantes sintomas psiquiátricos póstraumáticos ${ }^{18-24}$. Essas situações negativas na infância possuem alto grau de relação com sinais e sintomas comórbidos ao TEPT, traduzidos como queixas dissociativas, somatizações, alterações de atenção, consciência e autopercepção, impulsividade e alterações afetivas ${ }^{2,25}$.

Levando em conta que o período do nascimento até a adultez é marcado pelo progressivo desenvolvimento físico, comportamental e emocional ${ }^{26}$, exposições prolongadas a experiências traumáticas durante a infância ainda comportam uma preocupante realidade e podem produzir profundo impacto em diferentes áreas funcionais. Cabe ressaltar que, em algumas ocasiões, o efeito do trauma pode se apresentar através dos sintomas centrais de TEPT, como revivescência do trauma, esquiva/entorpecimento emocional e hiperexcitabilidade autonômica. Em outros momentos, como na exposição crônica a eventos traumáticos ou na contínua negligência por parte dos cuidadores, pode haver uma série de sintomas afetivos e interpessoais associados, ou mesmo um aumento da ocorrência de outras psicopatologias, entre elas, depres- são maior, transtorno de ansiedade generalizada, transtorno do pânico e uso de substâncias ${ }^{27-29}$.

Em termos de psicopatologia infantil, o TEPT não é o diagnóstico psiquiátrico mais comum em crianças com histórico de abuso e negligência ${ }^{30}$. Estes são, em ordem de frequência, transtorno de ansiedade de separação, transtorno desafiador opositivo, transtorno de fobia social, TEPT e déficit de atenção e hiperatividade (TDAH) ${ }^{31}$. Além disso, os critérios diagnósticos de TEPT são utilizados tanto para adultos como para crianças, apesar de o TEPT abranger apenas uma limitada parte dos sintomas apresentados pela população infantil.

Em mulheres adultas, o abuso sexual é a causa mais frequente de sintomas pós-traumáticos ${ }^{32}$. Entretanto, o número de mulheres que sofreu algum tipo de abuso sexual na infância é extremamente maior quando comparado ao número de abusos na idade adulta ${ }^{28}$. Além disso, indivíduos com histórico de abuso na infância raramente são expostos a apenas um único evento traumático, pois geralmente o abusador é alguém próximo da vítima, o que acaba por dificultar uma possível denúncia ${ }^{33,34}$. Sabe-se também que, em se tratando de violência doméstica, o trauma pode ser mantido em segredo por longo tempo, prejudicando a identificação de alguma síndrome pós-traumática.

Tal discussão teve início através de estudos longitudinais e da observação clínica, quando alguns autores questionaram o fato de abusos psicológicos crônicos na infância estarem altamente relacionados com a possibilidade de as vítimas futuramente desenvolverem psicopatologias ${ }^{14,35}$ ${ }^{37}$. Evidenciou-se que pacientes expostos a uma contínua carga de negligência apresentavam determinados sintomas, tais como: sintomas somáticos (dor de cabeça, distúrbios gastrintestinais, dores abdominais, lombares e pélvicas, tremores, sensações de choque e náusea), dissociativos (alteração da realidade, alteração de personalidade, alucinações, confusão temporal entre presente e passado) e afetivos (sintomas depressivos, insônia, apatia, desamparo, culpa, dificuldades de concentração, comportamentos suicidas, alteração na visão de si e dos outros). Salienta-se também a presença de hipervigilância, agitação e ansiedade extrema em consequência das recordações das memórias traumáticas ${ }^{38}$. Tais sintomas não estão inclusos no diagnóstico de TEPT vigente ${ }^{39}$.

As evidências descritas corroboraram a adoção do termo trauma complexo para descrever as consequências da exposição contínua e prolongada a eventos estressores de natureza interpessoal, como uma tentativa de melhor refletir a gravidade da sintomatologia associada ao trauma psicológico e desenvolvimental ${ }^{14,40}$.

\section{Disorders of extreme stress, not otherwise specified}

Como já mencionado, durante o desenvolvimento do DSMIV, a APA estabeleceu um comitê para a realização de uma pesquisa de campo. Os principais objetivos do estudo foram investigar 
possíveis mudanças nos critérios diagnósticos de TEPT e aprofundar o conhecimento sobre o desenvolvimento psicopatológico referente à exposição crônica a vivências traumáticas. Subdivididos em sete categorias sintomáticas, 27 sintomas não contemplados pelo diagnóstico de TEPT descrito no DSM-III foram identificados no estudo. As categorias são: a) alteração na habilidade de modular as emoções; b) alterações na identidade e senso de self; c) alteração no curso de consciência e memória; d) alterações na relação com o agressor; e) alterações nas relações com os outros; f) alterações nas condições físicas e nas condições médicas; e g) alterações no sistema de significados ${ }^{41}$.

Baseado em estudos preliminares ${ }^{14}$, o grupo propôs a criação de uma nova categoria diagnóstica (DESNOS), abrangendo a gama de sintomas verificados em populações vítimas de estresse desenvolvimental contínuo e confirmando a existência de uma complexa (des)adaptação ao trauma (Tabela 1$)^{25}$. É importante ressaltar que alguns autores utilizam o termo TEPT complexo (complex post-traumatic stress disorder) ao citar o diagnóstico de DESNOS.

De acordo com os resultados do estudo, além da criação de uma nova categoria diagnóstica, o grupo levantou outra hipótese: crianças abusadas ou negligenciadas, mulheres vítimas de violência doméstica ou abusos sexuais e sobreviventes de cativeiro ou campo de concentração poderiam apresentar os sintomas descritos nos critérios diagnósticos de DESNOS, porém não necessariamente se enquadrar nos critérios diagnósticos para TEPT ${ }^{42}$.
Em situações de cativeiro, o agressor geralmente se torna a pessoa mais poderosa e influente na vida da vítima, podendo até mesmo modificar o sistema de crenças e valores desta, de acordo com sua perspectiva. Porém, quanto mais as relações com o mundo externo se mantiverem fortes, menor será a força do autor sobre a vítima ${ }^{14}$. No abuso sexual ou negligência infantil, os distúrbios de relação afetiva se agravam ainda mais. A instabilidade nos relacionamentos parentais, a exposição precoce à violência e o provável convívio diário com o abusador resultam em alterações negativas na visão de mundo e nas relações interpessoais. Além disso, há prejuízos nas áreas de segurança, confiança, amor, intimidade, controle, juntamente com dificuldades específicas de tolerância, autorregulação afetiva, autoestima e incapacidade de ser benevolente com os outros ou de expressar afeto. Indivíduos que padecem de controle coesivo sofrem profundas alterações na sua própria visão de self, e isso se traduziria em sintomas psiquiátricos relacionados ${ }^{43}$.

Outra questão diz respeito ao tratamento para TEPT, pois o mesmo se foca prioritariamente nas memórias relacionadas ao evento traumático específico, a fim de diminuir seus efeitos negativos, dando ao paciente um domínio sobre seus medos através da ressignificação/extinção da memória traumática e do ensino de habilidades de manejo frente às situações temidas $^{44}$. Em contrapartida, o tratamento para DESNOS se focaria inicialmente no desajuste emocional e nos prejuízos das relações interpessoais ${ }^{45}$, em virtude da importância do manejo

Tabela 1 - Critérios diagnósticos do transtorno de estresse extremo sem outra especificação (disorders of extreme stress, not otherwise specified - DESNOS)

\begin{tabular}{ll}
\hline A. Alteração na regulação do afeto e impulsos & - Regulação afetiva \\
& - Modulação da raiva \\
& - Comportamento autodestrutivo \\
& - Ideação suicida \\
& - Dificuldade na modulação de comportamento sexual \\
& - Excessiva exposição ao risco \\
& - Amnésia \\
& - Episódios dissociativos transitórios e despersonalização \\
B. Alterações na atenção ou consciência & - Sistema digestório \\
& - Dor crônica \\
C. Somatização & - Sintomas cardiopulmonares \\
& - Sintomas de conversão \\
& - Sintomas sexuais \\
& - Ineficácia \\
& - Danos permanentes \\
& - Culpa e responsabilidade \\
& - Vergonha \\
D. Alterações na autopercepção & - Dificuldade de ser compreendido \\
& - Minimização \\
& - Adoção de crenças distorcidas \\
& - Idealização do agressor \\
& - Preocupação com o bem-estar do agressor \\
& - Incapacidade para confiar \\
& - Revitimização \\
E. Alterações na percepção do agressor & - Vitimizar outros \\
& - Desespero e desesperança \\
& - Perda de crenças fundamentais anteriores ao trauma \\
\hline &
\end{tabular}


da regulação afetiva e do fortalecimento dos vínculos sociais durante o tratamento de síndromes pós-traumáticas, que geralmente sofrem alterações negativas devido à exposição a estressores traumáticos ${ }^{33,42}$.

Entretanto, o comitê organizador do DSM-IV optou por considerar os sintomas descritos no DESNOS apenas como correlatos ao TEPT ${ }^{25}$. Posteriormente, a decisão de não incluir o DESNOS no DSM-IV resultou em pouca atenção dada aos problemas correlacionados ao TEPT, que muitas vezes fazem parte da gama central de sintomas pós-traumáticos.

Atualmente, estudos seguem investigando o constructo do DESNOS ${ }^{46}$, a existência de uma entrevista estruturada (Structured Interview for Disorders of Extreme Stress - SIDES) destinada à avaliação sintomática do $\operatorname{DESNOS}^{41}$ e a presença de muitas alterações descritas nesse diagnóstico no Manual de Classificação Internacional de Doenças e Problemas Relacionados à Saúde (CID-10), comumente na categoria de transtornos de personalidade, como diagnóstico de alterações permanentes de personalidade após experiência catastrófica. Tais iniciativas reforçam a relevância do DESNOS, consolidando o transtorno como possível categoria diagnóstica para a próxima versão do $\mathrm{DSM}^{47}$.

\section{Developmental trauma disorder}

Após a primeira concepção de uma categoria diagnóstica que abrangesse os efeitos do trauma complexo (DESNOS), estudos ratificaram que a exposição contínua a eventos traumáticos cada vez mais é associada a inúmeros efeitos negativos, relativos às áreas emocionais, comportamentais, cognitivas e sociais, principalmente em períodos críticos do desenvolvimento infantil ${ }^{42}$.

Em 2005, os integrantes de uma força-tarefa de trauma complexo (National Child Traumatic Stress Network) delinearam uma nova categoria diagnóstica, intitulada transtorno de desenvolvimento traumático (DTD). O diagnóstico proposto foi delineado em virtude de: a) desregulação emocional desencadeada nas respostas às memórias traumáticas; e b) generalizações de estímulo decorrentes do impacto do trauma complexo durante o desenvolvimento ${ }^{16}$.

Para a concepção do DTD, os pesquisadores analisaram estudos que se propuseram a investigar quadros clínicos póstraumáticos em crianças e adolescentes ${ }^{48}$, concluindo que crianças expostas a eventos traumáticos, como maus-tratos, negligência, abandono, violência doméstica, separação dos pais, perdas traumáticas ou comportamentos sexuais inapropriados, não preencheriam o critério A do DSM-IV necessário para o diagnóstico de TEPT. Entretanto, em termos de sintomas, essas crianças poderiam apresentar sintomas típicos de TEPT em conjunto com outros sintomas, ainda durante a infância ou futuramente, na adultez ${ }^{49}$. Tais sintomas associados incluiriam problemas na regulação emocional, drásticas mudanças de humor e no padrão comportamental, perda de autonomia, comportamento agressivo diante dos outros e de si mesmo, perda da regulação do sono, da fome e de autocuidados, múltiplos problemas somáticos (desde queixas gastrintestinais até dores de cabeça), aparente perda da avaliação e discriminação de estímulos ameaçadores (aumento dos comportamentos de risco), sentimentos de ódio, autoculpa e os crônicos sentimentos de impotência (Tabela 2) ${ }^{16}$.

Tabela 2 - Critérios diagnósticos do transtorno de desenvolvimento traumático (developmental trauma disorder - DTD)

\begin{tabular}{|c|c|}
\hline A. Exposição & $\begin{array}{l}\text { - Múltipla ou crônica exposição a uma ou mais formas de adversidades } \\
\text { desenvolvimentais de natureza traumática e interpessoal (abandono, traição, } \\
\text { agressões físicas, agressões sexuais, ameaças à integridade física, práticas } \\
\text { coercivas, abuso emocional, testemunhar violência e morte) } \\
\text { - Experiências subjetivas de raiva, traição, resignação, medo, derrota e vergonha }\end{array}$ \\
\hline $\begin{array}{l}\text { B. Desencadeamento de um padrão repetitivo de } \\
\text { desregulação frente a estímulos associados ao trauma }\end{array}$ & $\begin{array}{l}\text { - Caracteriza-se pela desregulação nas seguintes áreas: } \\
\text { - Afetiva } \\
\text { - Somática (fisiológica e motora) } \\
\text { - Comportamental } \\
\text { - Cognitiva (confusão, dissociação, despersonalização, revivescência) } \\
\text { - Relacional (conduta opositora, desconfiada, complacente) } \\
\text { - Autoatribuição (autoculpa e ódio a si próprio) }\end{array}$ \\
\hline C. Atribuições e expectativas persistentemente alteradas & $\begin{array}{l}\text { - Autoatribuição negativa } \\
\text { - Desconfiança do cuidador } \\
\text { - Perda da expectativa de proteção pelos outros } \\
\text { - Perda da confiança nos órgãos sociais de proteção } \\
\text { - Falta de recursos na busca de justiça/retribuição social } \\
\text { - Inevitabilidade de futura vitimização }\end{array}$ \\
\hline D. Prejuízo funcional & $\begin{array}{l}\text { - Educacional } \\
\text { - Familiar } \\
\text { - Interpessoal } \\
\text { - Vocacional } \\
\text { - Legal }\end{array}$ \\
\hline
\end{tabular}


Crianças expostas a contínuas vivências traumáticas de natureza interpessoal irão experimentar emoções intensas de raiva, medo, resignação, vergonha, sensação de derrota e traição ${ }^{35}$. Tais crianças desenvolvem certos comportamentos a fim de evitar ao máximo a recorrência dessas emoções. Porém, elas tendem a reviver os acontecimentos traumáticos atuando como perpetuadores de agressões ou de violência sexual contra outras crianças. Nessas crianças, o menor estímulo seria capaz de desencadear medo intenso; e, após esse estado de agitação, elas apresentariam extrema dificuldade em retornar à normalidade. Soma-se a isso o fato de que a expectativa de retorno do trauma permeia os relacionamentos das crianças vítimas de trauma complexo.

Tendo em vista que a exposição ao trauma durante o desenvolvimento infantil altera negativamente a resposta futura ao estresse ${ }^{50}$, essas crianças apresentam dificuldades em lidar com suas próprias emoções. Isso se expressa através de atribuições negativas a si mesmo, perda de confiança nas outras pessoas e descrença de que alguém vá cuidá-las, gerando sentimentos de insegurança. Sendo assim, essas crianças organizam seus relacionamentos em torno da expectativa ou da prevenção do abandono ou vitimização $0^{16}$.

Cabe salientar que estilos de apego durante a infância são fatores relevantes na qualidade do processo de informação durante a vida ${ }^{51}$. Crianças seguras aprendem um amplo vocabulário - amor, raiva, prazer, medo, ódio - para descrever suas emoções e comunicar o que sentem ${ }^{52}$. Por outro lado, crianças expostas a ambientes familiares conturbados, vítimas de abuso e maus-tratos, frequentemente apresentam dificuldades extremas para relatar o que sentem e expressar suas próprias emoções ${ }^{34}$.

Em um estudo de caso clínico, uma criança de 9 anos de idade foi diagnosticada com TEPT, TDAH, episódio depressivo de um transtorno bipolar, além de preencher os critérios para transtorno desafiador opositivo. De acordo com seu histórico, a mãe era portadora de transtorno esquizoafetivo e retardo mental. Retirada dos cuidados maternos aos 2 anos de idade, a criança passou a alternar entre morar na rua e viver em casas de acolhimento. Na maioria das vezes, ela era expulsa após pouco tempo, devido à conduta agressiva e comportamentos impulsivos. Seu histórico também relata exposição contínua a negligência e maus-tratos ${ }^{53}$.

Essa criança foi submetida a uma nova avaliação psicológica aos 12 anos de idade, desta vez feita por um especialista em trauma complexo, sendo então diagnosticada com DTD. Com isso, foi proposto um tratamento psicoterápico focado nos sintomas nucleares do DTD, associado a tratamento farmacológico. A recorrência de episódios agressivos foi reduzida, em conjunto com significativas melhoras nas relações sociais, tendo a paciente aprendido a enfrentar sintomas e ansiedades, o que evita o uso de comportamentos de risco ${ }^{53}$.

A associação entre múltiplos diagnósticos leva à utilização de psicofármacos de diferentes classes. Essas medicações podem causar diversos efeitos colaterais, como agi- tação, hiperatividade ou sedação, além de desviar o foco do tratamento do que seria a questão central: a história traumática do paciente. O tratamento focado no DTD possui metas principais de intervenção, dentre as quais é necessário, inicialmente, o estabelecimento de um ambiente seguro para o paciente, no qual não existam recorrências dos eventos traumáticos. $\mathrm{O}$ tratamento visa desenvolver as capacidades de modulação das emoções do paciente, ou seja, sua capacidade de retorno ao equilíbrio após excitação por algum estímulo interpretado como nocivo. Além disso, é trabalhado o controle da autorregulação do afeto e do comportamento, incluindo a reestruturação cognitiva ${ }^{54}$. Por fim, são realizadas intervenções associadas ao processamento das informações, desenvolvendo no paciente a capacidade de envolver processos de atenção no funcionamento cognitivo, como reestruturação do planejamento e tomada de decisão $0^{53}$.

A ressignificação e contenção da memória, o desenvolvimento de estratégias de enfrentamento e a orientação do pensamento e do comportamento para o tempo presente são estratégias terapêuticas utilizadas a fim de promover a integração das experiências traumáticas, através da transformação, incorporação e resolução das memórias relativas a essas vivências traumáticas. O reengajamento social é um ponto crucial no tratamento do DTD, com ênfase na restauração ou criação de novos laços afetivos. Desse modo, o desenvolvimento da aliança terapêutica e a empatia por parte do psicoterapeuta é fundamental para a capacidade do paciente de desenvolver reconfiança nas outras pessoas ${ }^{55}$. O reforço da autoestima e a autoavaliação positiva, em conjunto com o cultivo da criatividade pessoal, imaginação e orientação futura, são requisitos para o crescimento dos afetos positivos e da capacidade de sentir prazer. O desenvolvimento da assertividade, cooperação, fixação de limites, reciprocidade e capacidade de intimidade emocional são outras áreas desenvolvidas durante o tratamento ${ }^{53}$.

De acordo com a força-tarefa de trauma complexo, crianças traumatizadas precisam de auxílio qualificado para trabalhar a adaptação às lembranças dos eventos traumáticos, no sentido de ajudá-las a se focar no momento atual. É de responsabilidade do psicoterapeuta amparar essas crianças para que se sintam aptas a sentir prazer novamente. Devido ao intenso medo, essas crianças evitam estar engajadas em atividades e relações sociais por receio de que a iminência de um estímulo aversivo evoque lembranças traumáticas. Desse modo, faz-se necessário que o tratamento as ajude a se sentirem seguras a ponto de explorar o mundo e desenvolver sua curiosidade $^{16}$.

Questões referentes às possíveis alterações negativas no neurodesenvolvimento estão associadas ao conceito de trauma complexo, tornando-o fator etiológico para o desenvolvimento do DTD. Essa nova categoria compreende as implicações clínicas e diagnósticas das múltiplas vivências traumáticas desenvolvimentais de maneira mais completa e eficaz ${ }^{56}$. Tendo em vista que algumas crianças vítimas de exposição a estressores crônicos não preenchem os critérios 
diagnósticos para TEPT, inúmeros diagnósticos são utilizados na tentativa de abranger os sintomas apresentados por essas crianças e adolescentes ${ }^{39}$.

\section{Conclusões}

Entre os indivíduos expostos a múltiplas experiências traumáticas, ressalta-se que, além dos sintomas descritos no diagnóstico de TEPT, é comum o aparecimento de sintomas correlatos que não seriam adequadamente classificados pelo DSM-IV. O agravamento desses sintomas está associado ao número de experiências traumáticas, bem como à gravidade desses traumas. Evidencia-se que essas marcas traumáticas diferem, no que tange à resposta humana ao estresse crônico, das respostas descritas no diagnóstico do TEPT. A sintomatologia identificada nas vítimas de exposição crônica ao trauma começa a ser corretamente descrita a partir dos estudos dedicados à criação de categorias diagnósticas referentes ao conceito de trauma complexo ${ }^{57}$. Desse modo, a atual ausência de um diagnóstico coeso e fidedigno para essas vítimas interfere negativamente na identificação sintomatológica e na seleção das intervenções terapêuticas. Devido ao grande número de crianças vítimas de violência, há uma disparidade entre a atual demanda e os métodos de tratamento disponívei ${ }^{58}$.

Outro ponto relevante é o fato de alguns estudos estarem considerando a possibilidade de criação de uma nova categoria de transtornos do eixo I no DSM-V. Denominada de transtornos de estresse traumático, ela abrangeria os diagnósticos de TEPT, o transtorno de estresse agudo, o transtorno de ajustamento e alguma categoria referente às síndromes associadas ao trauma complexo ${ }^{59}$.

Atualmente, há um consenso na comunidade científica de que a precoce exposição ao estresse afeta aspectos neuroimunopsicobiológicos desenvolvimentais, desencadeando futuras psicopatologias ${ }^{24}$. Um recente estudo avaliou alterações neuroestruturais em vítimas de abuso infantil diagnosticadas com TEPT complexo $(n=31)$, comparando esses pacientes a um grupo de controles saudáveis $(\mathrm{n}=28)$. De acordo com os resultados, as vítimas de TEPT complexo apresentaram diminuição de volume de massa cinzenta no giro do córtex cingulado anterior, além de redução na concentração de substância cinzenta no córtex órbito-frontal direito. Tais achados sugerem que indivíduos expostos a trauma complexo desenvolvem alterações críticas na estrutura cerebral, de maior prejuízo em comparação com as alterações neurológicas descritas na literatura para TEPT ${ }^{60}$.

A ideia apresentada neste artigo, referente aos sintomas associados à múltipla exposição a experiências traumáticas, é de que eles não constituiriam sintomas de comorbidades diagnósticas, e sim fariam parte de uma síndrome com características sintomatológicas somáticas, afetivas e comportamentais específicas relacionadas a traumas precoces. Portanto, manifestações negativas decorrentes do trauma interpessoal crônico fariam parte de uma doença primária.
Acredita-se que um novo diagnóstico é necessário para corretamente classificar essas situações, dando conta de suas consequências e sintomas. Dessa maneira seria possível uma definição clara dos efeitos do trauma durante o desenvolvimento $^{12}$. A inclusão do DTD na próxima versão do DSM provavelmente não irá ocorrer, apesar de o comitê estar estudando essa possibilidade. Provavelmente, esse transtorno entrará como um conjunto de critérios e eixos propostos para estudos adicionais na nova edição do manual.

Apesar de incipiente, acredita-se que a disseminação de estudos em trauma complexo fomentará o desenvolvimento contínuo dos diagnósticos de DESNOS e DTD. Para isso, será necessária a integração dos campos da psicopatologia do desenvolvimento e das neurociências. Além disso, há uma carência de mais pesquisas de validação de constructo e validação transcultural. É necessário, também, o desenvolvimento e adaptação de mais instrumentos de avaliação associados ao trauma complexo, bem como uma precisa identificação dos componentes terapêuticos relevantes à recuperação dos pacientes traumatizados.

\section{Referências}

1. American Psychiatric Association, Task Force on DSM-IV. Diagnostic and Statistical Manual of Mental Disorders: DSM-IV-TR Fourth Edition. Washington: American Psychiatric Association; 2000.

2. Brewin C, Lanius R, Novac A, Schnyder U, Galea S. Reformulating PTSD for DSM-V: life after criterion A. J Trauma Stress. 2009;22(5):366-73.

3. Weathers F, Keane T. The criterion A problem revisited: controversies and challenges in defining and measuring psychological trauma. J Trauma Stress. 2007;20(2):107-21.

4. Kilpatrick DG, Resnick HS, Acierno R. Should PTSD criterion A be retained? J Trauma Stress. 2009;22(5):374-83

5. Van der Kolk BA, McFarlane AC, Weisæth L. Traumatic stress: the effects of overwhelming experience on mind, body, and society. New York: Guilford Press; 1996.

6. Roberts J, deRoon-Cassini T, Brasel K. Posttraumatic stress disorder: a primer for trauma surgeons. J Trauma. 2010;69(1):231-7.

7. Solomon Z, Horesh D. Changes in diagnostic criteria of PTSD: implications from two prospective longitudinal studies. Am J Orthopsychiatry. 2007;77(2):182-8.

8. Andreasen N. Acute and delayed posttraumatic stress disorders: a history and some issues. Am J Psychiatry. 2004;161(8):1321-3.

9. Grassi-Oliveira R. Balzacian PTSD: diagnostic considerations. Rev Psiquiatr Rio Gd Sul. 2007;29(3):333-4.

10. Palmiere P, ed. Factor structure of PTSD: implications for DSM-V. In: 24th ISTSS Annual Meeting - Terror and its Aftermath; 2008; Chicago, USA.

11. Friedman M, Brewin C, Bryant R, Keane T, Kilpatrick D, Schnurr P, eds. Considering PTSD for DSM-V. In: 25th ISTSS Annual Meeting - Traumatic Stress Disorders: Toward DSM-V and ICD-11; 2009; Atlanta, USA.

12. van der Kolk BA, Courtois CA. Editorial comments: complex developmental trauma. J Trauma Stress. 2005;18(5):385-8.

13. Cloitre M, ed. What is complex about complex PTSD and does it matter for treatment? In: 24th ISTSS Annual Meeting - Terror and its Aftermath; 2008; Chicago, USA.

14. Herman JL. Complex PTSD: a syndrome in survivors of prolonged and repeated trauma. J Trauma Stress. 1992;5(3):377-91.

15. van der Hart O, Nijenhuis ERS, Steele K. Dissociation: an insufficiently recognized major feature of complex posttraumatic stress disorder. J Trauma Stress. 2005;18(5):413-23.

16. van der Kolk B. Developmental trauma disorder. Psychiatr Ann. 2005;35(5):401-8.

17. Taylor S, Asmundson G, Carleton R. Simple versus complex PTSD: a cluster analytic investigation. J Anxiety Disord. 2006;20(4):459-72.

18. McEwen BS. Physiology and neurobiology of stress and adaptation: central role of the brain. Physiol Rev. 2007;87(3):873-904. 
19. Weiss SJ. Neurobiological alterations associated with traumatic stress. Perspect Psychiatr Care. 2007;43(3):114-22.

20. Raone A, Cassanelli A, Scheggi S, Rauggi R, Danielli B, De Montis MG. Hypothalamus-pituitary-adrenal modifications consequent to chronic stress exposure in an experimental model of depression in rats. Neuroscience. 2007;146(4):1734-42.

21. McEwen B. The brain is the central organ of stress and adaptation. Neuroimage. 2009;47(3):911-3.

22. McGowan PO, Sasaki A, DAlessio AC, Dymov S, Labonte B, Szyf M, et al Epigenetic regulation of the glucocorticoid receptor in human brain associates with childhood abuse. Nat Neurosci. 2009;12(3):342-8.

23. Grassi-Oliveira R, Stein L. Childhood maltreatment associated with PTSD and emotional distress in low-income adults: the burden of neglect. Child Abuse Negl. 2008;32(12):1089-94

24. Shonkoff J, Boyce W, McEwen B. Neuroscience, molecular biology, and the childhood roots of health disparities: building a new framework for health promotion and disease prevention. JAMA. 2009;301(21):2252-9.

25. van der Kolk BA, Roth S, Pelcovitz D, Sunday S, Spinazzola J. Disorders of extreme stress: the empirical foundation of a complex adaptation to trauma. J Trauma Stress. 2005;18(5):389-99.

26. Grassi-Oliveira R, Ashy M, Stein L. Psychobiology of childhood maltreatment: effects of allostatic load? Rev Bras Psiquiatr. 2008;30(1):60-8.

27. Roestel C, Kersting A. [Simple and complex post-traumatic stress disorders. Diagnostic and therapeutic approaches]. Nervenarzt. 2008;79(7):845-54; quiz 55.

28. Breslau N, Davis G, Andreski P, Peterson E, Schultz L. Sex differences in posttraumatic stress disorder. Arch Gen Psychiatry. 1997;54(11):1044-8.

29. Cloitre M, Cohen L, Edelman R, Han H. Posttraumatic stress disorder and extent of trauma exposure as correlates of medical problems and perceived health among women with childhood abuse. Women Health. 2001;34(3):1-17.

30. Putnam F. Ten-year research update review: child sexual abuse. J Am Acad Child Adolesc Psychiatry. 2003;42(3):269-78.

31. Ackerman P, Newton J, McPherson W, Jones J, Dykman R. Prevalence of post traumatic stress disorder and other psychiatric diagnoses in three groups of abused children (sexual, physical, and both). Child Abuse Negl. 1998;22(8):759-74.

32. Kessler R, Sonnega A, Bromet E, Hughes M, Nelson C. Posttraumatic stress disorder in the National Comorbidity Survey. Arch Gen Psychiatry. 1995;52(12):1048-60.

33. Cloitre M, Stovall-McClough K, Miranda R, Chemtob C. Therapeutic alliance, negative mood regulation, and treatment outcome in child abuse-related posttraumatic stress disorder. J Consult Clin Psychol. 2004;72(3):411-6.

34. Cloitre M, Stolbach B, Herman J, Kolk B, Pynoos R, Wang J, et al. A developmental approach to complex PTSD: childhood and adult cumulative trauma as predictors of symptom complexity. J Trauma Stress. 2009 Sep 30. [Epub ahead of print]

35. Perepletchikova F, Kaufman J. Emotional and behavioral sequelae of childhood maltreatment. Curr Opin Pediatr. 2010;22(5):610-5.

36. Kim K, Trickett P, Putnam F. Childhood experiences of sexual abuse and later parenting practices among non-offending mothers of sexually abused and comparison girls. Child Abuse Negl. 2010;34(8):610-22.

37. Chen L, Murad M, Paras M, Colbenson K, Sattler A, Goranson E, et al. Sexual abuse and lifetime diagnosis of psychiatric disorders: systematic review and meta-analysis. Mayo Clin Proc. 2010;85(7):618-29.

38. Herman J, Harvey M. Adult memories of childhood trauma: a naturalistic clinical study. J Trauma Stress. 1997;10(4):557-71.

39. Fehrnerback T, Kisiel C, Griffin G, Roberts L, Marett J, eds. Implications of developmental trauma disorder for children in child welfare. In: 25th ISTSS Annual Meeting - Traumatic Stress Disorders: Toward DSM-V and ICD-11; 2009; Atlanta, USA.
40. Kisiel C, Ghosh Ippen C, Burkman K, DAndrea W, Cloitre M, eds. Cumulative trauma in childhood. In: 25th ISTSS Annual Meeting - Traumatic Stress Disorders: Toward DSM-V and ICD-11; 2009; Atlanta, USA.

41. Pelcovitz D, van der Kolk B, Roth S, Mandel F, Kaplan S, Resick P. Development of a criteria set and a structured interview for disorders of extreme stress (SIDES). J Trauma Stress. 1997;10(1):3-16.

42. van der Kolk B, Roth S, Pelcovitz D, Sunday S, Spinazzola J. Disorders of extreme stress: the empirical foundation of a complex adaptation to trauma. J Trauma Stress. 2005;18(5):389-99.

43. Pearlman L, Courtois C. Clinical applications of the attachment framework: relational treatment of complex trauma. J Trauma Stress. 2005;18(5):449-59.

44. Holmes E, Moulds M, Kavanagh D. Memory suppression in PTSD treatment? Science. 2007;318(5857):1722; author reply 1722

45. Ford J, Courtois C, Steele K, Hart O, Nijenhuis E. Treatment of complex posttraumatic self-dysregulation. J Trauma Stress. 2005;18(5):437-47.

46. Dorahy M, Corry M, Shannon M, Macsherry A, Hamilton G, McRobert G, et al. Complex PTSD, interpersonal trauma and relational consequences: findings from a treatment-receiving Northern Irish sample. J Affect Disord. 2009;112(1-3):71-80.

47. American Psychiatric Association. DSM-V Prelude Project. Arlington: APA; 2010. Disponível em: www.dsm5.org. Acessado 7 out 2010.

48. Simons M, Herpertz-Dahlmann B. [Trauma and traumatic disorders in children and adolescents]. Z Kinder Jugendpsychiatr Psychother. 2008;36(3):151-61.

49. van der Kolk B, ed. Clinical and research implications of developmental trauma disorder. In: 25th ISTSS Annual Meeting - Traumatic Stress Disorders: Toward DSM-V and ICD-11; 2009; Atlanta, USA.

50. Anda R, Felitti V, Bremner J, Walker J, Whitfield C, Perry B, et al. The enduring effects of abuse and related adverse experiences in childhood. A convergence of evidence from neurobiology and epidemiology. Eur Arch Psychiatry Clin Neurosci. 2006;256(3):174-86.

51. Dong M, Anda R, Felitti V, Dube S, Williamson D, Thompson T, et al. The interrelatedness of multiple forms of childhood abuse, neglect, and household dysfunction. Child Abuse Negl. 2004;28(7):771-84.

52. Dorard G, Berthoz S, Phan O, Corcos M, Bungener C. Affect dysregulation in cannabis abusers: a study in adolescents and young adults. Eur Child Adolesc Psychiatry. 2008;17(5):274-82.

53. Levin $\mathrm{E}$. The challenges of treating developmental trauma disorder in a residential agency for youth. J Am Acad Psychoanal Dyn Psychiatry. 2009;37(3):519-38.

54. Schmid M, Goldbeck L. [Cognitive behavioral therapy for adolescents suffering from complex trauma disorder]. Prax Kinderpsychol Kinderpsychiatr. 2010;59(6):453-76

55. Cohen J, Mannarino A. Psychotherapeutic options for traumatized children. Curr Opin Pediatr. 2010;22(5):605-9.

56. Nader K, Fletcher K, Ford J, Briere J, Pelcovitz D, van der Kolk B, eds. Complex trauma in children and adolescents: conceptualization and assessment. In: 24th ISTSS Annual Meeting - Terror and its Aftermath; 2008; Chicago, USA.

57. Allen J, Coyne L, Huntoon J. Complex posttraumatic stress disorder in women from a psychometric perspective. J Pers Assess. 1998;70(2):277-98.

58. Ramiro L, Madrid B, Brown D. Adverse childhood experiences (ACE) and health-risk behaviors among adults in a developing country setting. Child Abuse Negl. 2010;34(11):842-55.

59. Miller M, Resick P, Keane T. DSM-V: should PTSD be in a class of its own? Br J Psychiatry. 2009;194(1):90.

60. Thomaes K, Dorrepaal E, Draijer N, de Ruiter M, van Balkom A, Smit J, et al. Reduced anterior cingulate and orbitofrontal volumes in child abuse-related complex PTSD. J Clin Psychiatry. 2010;71(12):1636-44. 